\section{PET for the Evaluation of Solitary Pulmonary Nodules}

TO THE EDITOR: The recent article by Fletcher et al. (1) reasonably shows, as the authors claim, that PET is more accurate than CT in evaluating solitary pulmonary nodules. The data presented in the article, however, also address an even more controversial clinical issue, namely: what is the visual or standardized uptake value (SUV) cutoff for classifying a solitary pulmonary nodule as benign? The traditional teaching, supported by numerous early, pivotal studies, including several by the authors of this paper, is that nodules with an SUV less than 2.5 or whose activity appears visually to be less than or equal to that of the mediastinal blood pool can be considered benign with enough confidence to avoid an immediate biopsy; these nodules can safely be followed with CT $(2,3)$. Recently, several groups have published data contradicting this principle (4), including evidence that any visually perceptible uptake by a pulmonary nodule is associated with a significant chance of malignancy (5). This contradictory evidence has left modern readers of PET scans in a quandary-how does one classify a pulmonary nodule with detectable, but low, tracer uptake? Many readers, influenced either by the recent articles or by their own data obtained by careful follow-up of their scans, have discarded the traditional criteria and interpret as benign only those nodules completely negative on PET. Others, perhaps the majority, continue to use the original criteria. The data of Fletcher et al. seem well suited to finally eliminating the original criteria once and for all. The data are all included in Table 3 but can be slightly rearranged statistically to best address the problem using negative predictive values (NPVs. NPV is of course dependent on the prevalence of disease in the study population, but the authors reported that their prevalence of malignancy $(53 \%)$ was lower than in comparable studies, which actually strengthens the arguments made in this letter.):

NPV of a clearly benign nodule (no uptake $)=97 \%$

NPV of a probably benign nodule $(\mathrm{SUV}<1.5-2.0)=87 \%$

$\mathrm{NPV}$ of an indeterminate nodule $(\mathrm{SUV}=1.5-2.0)=78 \%$

Certainly, everyone would accept a $97 \%$ probability of benign as good enough to avoid a biopsy and to follow with CT. But what about $87 \%$ ? I think a lot of physicians and patients would be reluctant to avoid a biopsy when there is a $13 \%$ chance of malignancy. The authors avoid this question by combining the first 2 groups of nodules, which leads to a combined NPV of $91 \%$, but this clouds the issue of what the visual or SUV cutoff should be. And finally, the data clearly show that nodules with uptake a little less than blood pool (SUV $=1.5-2.0$ ) carry a $22 \%$ chance of malignancy - much too high to avoid a biopsy. The traditional SUV cutoff of 2.5 is therefore clearly inadequate.

COPYRIGHT @ 2009 by the Society of Nuclear Medicine, Inc.
The question of exactly what probability of malignancy is safe enough to avoid a biopsy is quite complicated medically and ethically. What is not complicated, however, is the fact that these data, and those of other recent publications, unmistakably show that the traditional criteria for a benign nodule, namely activity less than or equal to mediastinal blood pool or SUV less than 2.5, are patently incorrect and should be discarded. Nuclear medicine physicians and radiologists are routinely asked by referring clinicians if their patients' nodules are very likely benign. Do the authors believe that only those nodules without detectable uptake should be interpreted as such, or could very low uptake, perhaps an SUV less than 1.5, be used as a cutoff?

\section{REFERENCES}

1. Fletcher JW, Kymes SM, Gould M, et al. A Comparison of the diagnostic accuracy of ${ }^{18} \mathrm{~F}$-FDG PET and CT in the characterization of solitary pulmonary nodules. J Nucl Med. 2008;49:179-185.

2. Duhaylongsod FG, Lowe VJ, Patz EF Jr, Vaughn AL, Coleman RE, Wolfe WG. Detection of primary and recurrent lung cancer by means of F-18 fluorodeoxyglucose positron emission tomography (FDG PET). J Thorac Cardiovasc Surg. 1995; 110:130-139.

3. Lowe VJ, Hoffman JM, DeLong DM, Patz EF, Coleman RE. Semiquantitative and visual analysis of FDG-PET images in pulmonary abnormalities. J Nucl Med. 1994;35:1771-1776.

4. Kim SK, Allen-Auerbach M, Goldin J, et al. Accuracy of PET/CT in characterization of solitary pulmonary lesions. J Nucl Med. 2007;48:214-220.

5. Hashimoto Y, Tsujikawa T, Kondo C, et al. Accuracy of PET for diagnosis of solid pulmonary lesions with ${ }^{18} \mathrm{~F}$-FDG uptake below the standardized uptake value of 2.5. J Nucl Med. 2006;47:426-431.

\author{
Ronald E. Fisher \\ Baylor College of Medicine \\ Houston, Texas
}

DOI: 10.2967/jnumed.108.054031

REPLY: Dr. Fisher makes several important points in his letter to the editor regarding the findings in our publication on ${ }^{18} \mathrm{~F}-\mathrm{FDG}$ PET in evaluation of solitary pulmonary nodules (1). As with any diagnostic test, PET in this application will be most useful when it is likely to change or modify the management approach. The ability of the test to do this depends on the likelihood of disease before the test- the disease prevalence - and the test performance characteristics. These factors then determine how we should decide who could benefit from a PET study and once the test has been performed how the result could influence management. Given the excellent performance parameters that we identified-a likelihood ratio of 9.9 for a positive PET read as "definitely malignant" and a likelihood ratio of 0.03 for a negative PET read as "definitely benign"-it is still unlikely that patients with either a very low $(5 \%)$ or very high pretest probability $(>80 \%)$ of a malignant solitary pulmonary nodule would benefit from this examination (2). In a cost-effectiveness analysis of ${ }^{18}$ F-FDG PET in solitary pulmonary nodules, Gould et al. reached similar conclusions and indicated that ${ }^{18}$ F-FDG PET should be used selectively when pretest probability and CT findings are discordant or in patients 
with intermediate pretest probability who are at high risk for surgical complications (3). They note importantly that the costeffectiveness of various diagnostic strategies depends critically on the pretest probability of malignancy.

The strength of the evidence required before a management decision is made will vary depending on the pretest likelihood of disease and the risk of a specific intervention. As Dr. Fisher indicates, a negative predictive value of a nodule with no uptake (i.e., "definitely benign" by our criteria) is $97 \%$ and is probably acceptable for adopting a watch-and-wait strategy, but a negative predictive value of a "probably benign nodule" (estimated standardized uptake value $>0.6-0.8$ but $<1.5-2.0$ ) is $87 \%$ and may not be convincing enough to avoid a biopsy, especially in a patient with a smoking history and other risk factors for malignancy (2). Although we dichotomized the 5 confidence levels of interpretation as described for determining sensitivity and specificity, we did develop interval likelihood ratios for each level of interpretation. In this regard, with our prevalence rate of $53 \%$ malignant nodules, a patient whose nodule was rated definitely benign by PET had a posttest probability of malignancy of only $3 \%$ as pointed out by Dr. Fisher. Similarly, a patient whose nodule was rated probably benign by PET had a posttest probability of $13 \%$. In populations with lower prevalence rates, the pretest-posttest probability decrease would be shifted even further. For example, in a population with a $20 \%$ prevalence of malignancy, the posttest probabilities would be reduced to $1 \%$ and $4 \%$ in patients with definitely benign and probably benign interpretations, respectively.

We strongly agree with Dr. Fisher about the hazards of continuing to consider a binary cutoff of 2.5 for standardized uptake value as capable of reliably distinguishing benign from malignant nodules. We would instead encourage the adoption of a visual scoring methodology with a validated, more continuous scale that relates to interval likelihood ratios, such as described in our publication. In this manner, the clinical pretest likelihood of malignancy could be incorporated into the final estimate of the posttest likelihood of a malignant or benign nodule.

\section{REFERENCES}

1. Fletcher JW, Kymes, Gould M, et al. A comparison of the diagnostic accuracy of ${ }^{18} \mathrm{~F}$-FDG PET and CT in the characterization of solitary pulmonary nodules. J Nucl Med. 2008;49:179-185.

2. Detterbeck FC, Falen S, Rivera MP, et al. Seeking a home for a PET, part 1: defining the appropriate place for positron emission tomography imaging in the diagnosis of pulmonary nodules or masses. Chest. 2004;125:2294-2299.

3. Gould MK, Sanders GD, Barnett PG, et al. Cost-effectiveness of alternative management strategies for patients with solitary pulmonary nodules. Ann Intern Med. 2003;138:724-735.

James W. Fletcher, for the VA SNAP Cooperative Studies Group Indiana/Purdue University at Indianapolis Indianapolis, Indiana

DOI: 10.2967/jnumed.108.056093

\section{Pregnancy Outcome After ${ }^{131}$ I Therapy}

TO THE EDITOR: We read with interest the article by Garsi et al. (1) concerning the pregnancy outcome and the health of offspring of women who had received ${ }^{131}$ I for differentiated thyroid cancer. In this article, the authors evaluated 2,673 pregnancies from patients treated with ${ }^{131} \mathrm{I}$ and found $10.4 \%$ miscarriages before any treatment, $20 \%$ after thyroidectomy but before ${ }^{131} \mathrm{I}$ therapy, and $19 \%$ after ${ }^{131}$ I therapy. There was no significant variation according to the cumulative ${ }^{131} \mathrm{I}$ dose. The incidences of stillbirths, preterm births, low birth weight, and congenital malformations were not significantly different before and after ${ }^{131} \mathrm{I}$ therapy. The authors concluded that there was no evidence that radioiodine therapy affected the outcomes of subsequent pregnancies and offspring.

Interestingly, we have reported a relatively similar study in a smaller number of patients. Our study predominantly examined the effect of ${ }^{131}$ I therapy $(3,700 \mathrm{MBq})$ on menstrual cycle or pregnancy in women less than $40 \mathrm{y}$ old. Specifically, we evaluated 45 women with differentiated thyroid cancer who were treated with ${ }^{131}$ I therapy and compared with 83 age-matched control women. We found menstrual cycle irregularities in $13.3 \%$ of patients before ${ }^{131} \mathrm{I}$ therapy but $31.1 \%$ after treatment. However, after ${ }^{131}$ I therapy there were no subsequent pregnancy abnormalities such as premature births, miscarriages, or congenital abnormalities in the 7 children who were borne of 6 of the 45 patients (2). Another study, of 49 pregnancies from 76 patients who received ${ }^{131}$ I therapy, found $10 \%$ miscarriages, $18 \%$ induced abortions, and no congenital malformations or first-year mortality (3). All these findings concur that ${ }^{131} \mathrm{I}$ therapy is safe regarding subsequent pregnancy outcome. However, our results suggest an increased incidence of menstrual cycle abnormalities after ${ }^{131} \mathrm{I}$ therapy. It will be interesting to see if Garsi et al. (1), in their large cohort of patients, noticed any such abnormalities induced by ${ }^{131} \mathrm{I}$ therapy.

\section{REFERENCES}

1. Garsi J-P, Schlumberger M, Rubino C, et al. Therapeutic administration of ${ }^{131} \mathrm{I}$ for differentiated thyroid cancer: radiation dose to ovaries and outcome of pregnancies. J Nucl Med. 2008;49:845-852.

2. Sioka C, Kouraklis G, Zafirakis A, et al. Menstrual cycle disorders after therapy with iodine-131. Fertil Steril. 2006;86:625-628.

3. Balenović A, Vlasić M, Sonicki Z, et al. Pregnancy outcome after treatment with radioiodine for differentiated thyroid carcinoma. Coll Antropol. 2006;30:743-748.

\section{Chrissa Sioka} Andreas Fotopoulos University Hospital of Ioannina Ioannina, Greece

DOI: 10.2967/jnumed.108.055020

REPLY: We were interested to see the letter of Chrissa Sioka and Andreas Fotopoulos about our article (1). In addition to reporting results similar to ours, showing that ${ }^{131} \mathrm{I}$ therapy is safe regarding subsequent pregnancy outcome, with no increase in the risk of miscarriage, induced abortion, or congenital malformation, they added new data showing that ${ }^{131}$ I therapy probably increases the incidence of menstrual cycle abnormalities (2).

To confirm these results, we analyzed the responses given by women in our series to similar questions. Of 2,190 women questioned about cycle abnormalities before and after their cancer and followed at least $2 \mathrm{y}$, we excluded 36 in whom another cancer had developed before thyroid cancer, 158 in whom another malignancy later developed, 263 who received external radiotherapy for thyroid cancer, and 137 who were treated with radioiodine for distant metastases. Of the remaining 1,866 women, 1,054 were 